\title{
Current clinical uses of the biomarkers for hepatocellular carcinoma
}

\author{
Jiwei Huang, Yong Zeng* \\ Department of Liver Surgery, Division of Liver Transplantation, West China Hospital, Sichuan University, Chengdu, Sichuan, China.
}

\begin{abstract}
Summary Hepatocellular carcinoma $(\mathrm{HCC})$ is a severe condition that is found worldwide. The current methods of HCC screening and diagnosis depend mainly on tumor imaging techniques. Using tumor biomarkers to detect cancer has helped to screen for disease and avoid wasting medical resources. Serum $\alpha$-fetoprotein (AFP), a glycoform of AFP that reacts with Lens culinaris agglutinin (AFP-L3), and des-gamma carboxyprothrombin (DCP) are biomarkers commonly used to detect $\mathrm{HCC}$ in medical practice around the world. However, each of these biomarkers is imperfect when used alone and each has limitations in terms of the sensitivity and specificity with which it detects HCC. Presumably, a combination of these biomarkers is a practical way to improve their performance. That said, novel biomarkers of $\mathrm{HCC}$ are being sought to diagnose the disease and also to optimize the treatment modality, to predict prognosis or recurrence, and to discover novel targets for therapeutic interventions.
\end{abstract}

Keywords: HCC, biomarkers, AFP, AFP-L3, DCP

Hepatocellular carcinoma (HCC) is one of the most common liver neoplasms in the world, with an estimated global incidence of over 700,000 new cases per year. It is prevalent in Asia and Africa, and its prevalence is increasing in the US, Canada, and Europe (1). The molecular pathogenesis of HCC is a complex multistep process in which many signaling cascades are altered, leading to a heterogeneous molecular profile. The management of HCC, including screening, diagnosis, therapy, and preventing recurrence, still poses numerous challenges. Current methods of HCC screening and diagnosis depend mainly on tumor imaging techniques, and especially ultrasonography. Surgical resection and liver transplantation are most promising therapies that offer the best therapeutic outcomes for treatment of HCC, with a 5-year overall survival rate of more than $50 \%$, but they are only feasible for early and intermediate-stage intervention (2).

Tumor biomarkers are types of molecules or substances that are objectively measurable in cells, tissues, or body fluids and that indicate the presence or predict the prognosis of tumors. Although the use of

\footnotetext{
*Address correspondence to:

Dr. Yong Zeng, Department of Liver Surgery, Division of Liver Transplantation, West China Hospital, Sichuan University, Chengdu, Sichuan, China.

E-mail: zengyongmd@gmail.com
}

serum biomarkers differs in countries in the East and in the West, these biomarkers augment radiological modalities in the current clinical treatment of HCC. The most widely used biomarker of HCC is serum $\alpha$-fetoprotein (AFP). Serum AFP is commonly regarded as a supplementary parameter for diagnosis and a tool for predicting recurrence and survival. AFP has been found to have a sensitivity of $41-65 \%$ and a specificity of 80 $90 \%$ when detecting HCC, given an AFP cut-off of 20 $\mathrm{ng} / \mathrm{mL}$ (3). A glycoform of AFP that reacts with Lens culinaris agglutinin (AFP-L3) is a second biomarker of HCC. AFP levels are only definitive once a tumor is large in size or in an advanced state, so AFP-L3 is used. Since AFP-L3 is more sensitive and specific for HCC than AFP alone, AFP-L3 is usually assayed with AFP. Des-gamma carboxyprothrombin (DCP) is another well-known biomarker for HCC. Also known as PIVKA II, or protein induced by vitamin $\mathrm{K}$ absence/antagonist- II, DCP is an abnormal prothrombin resulting from defective posttranslational carboxylation of the prothrombin precursor. Serum DCP detects HCC with varying levels of success according to studies. DCP is more likely to be elevated in patients with more advanced HCCs (e.g. larger tumors, vascular invasion, or metastasis).

Technological advances in genomics, proteomics, and metabolomics have enabled the discovery of novel HCC biomarkers. These newly discovered biomarkers can be roughly divided into the following categories: oncofetal and glycoprotein antigens (including AFP-L3 
Table 1. Clinical uses of the established biomarkers of HCC

Clinical uses Biomarkers

Screening AFP, AFP-L3, DCP

Diagnosis AFP, AFP-L3, DCP, GPC3, AFU, GGT,TSGF, TGF- $\beta$

Predicting mRNAs, HGF/SF, EGFR, human carbonyl reductase 2

prognosis

and GPC3), enzymes and isoenzymes (including DCP, GGT, AFU, and Human Carbonyl Reductase 2), growth factors and their receptors (including TGF- $\beta$, TSGF, EGFR, and HGF/SF), molecular markers (mRNAs), and pathological biomarkers. Clinical uses of these established HCC biomarkers are shown in Table 1. Unfortunately, no single biomarker can adequately provide a sufficient level of sensitivity and specificity for HCC. A combination of these biomarkers is a practical way to improve their performance. A combined assay of AFP with AFPL3 or des-g-carboxyprothrombin (DCP), which is widely used in Asia and especially in Japan, has better performance than either biomarker alone (4). The current study and use of biomarkers is far from comprehensive. Novel biomarkers of HCC could be used to screen for the disease and potentially helpful to optimize the treatment modality, to predict prognosis or recurrence, and to lead to novel targets for therapeutic interventions in the near future.

\section{References}

1. Ferenci P, Fried M, Labrecque D, et al. Hepatocellular carcinoma (HCC): A global perspective. J Clin Gastroenterol. 2010; 44:239-245.

2. Llovet JM, Bruix J. Novel advancements in the management of hepatocellular carcinoma in 2008. J Hepatol. 2008; 48 (Suppl 1):S20-37.

3. Daniele B, Bencivenga A, Megna AS, Tinessa V. Alpha-fetoprotein and ultrasonography screening for hepatocellular carcinoma. Gastroenterology. 2004; 127 (Suppl 1):S108-112.

4. Chaiteerakij R, Addissie BD, Roberts LR. Update on biomarkers of hepatocellular carcinoma. Clin Gastroenterol Hepatol. 2013; S1542-3565(13)01789-8.

(Received March 12, 2014; Revised April 17, 2014; Accepted April 21, 2014) 